\title{
Comparison of coronary computed tomography angiography image quality with high- and low-concentration contrast agents (CONCENTRATE): study protocol for a randomized controlled trial
}

Dong Jin Im², Yun-Hyeon Kim², Ki Seok Choo ${ }^{3}$, Joon-Won Kang ${ }^{4}$, Jung Im Jung ${ }^{5}$, Yoodong Won ${ }^{6}$, Hyo Rim Kim Myung Hee Chung ${ }^{8}$, Kyunghwa Han ${ }^{1}$ and Byoung Wook Choi ${ }^{1, *^{*}}$ (D)

\begin{abstract}
Background: With the development of computed tomography $(C T)$ technology, coronary $C T$ angiography can be acquired with low doses of radiation and contrast agent without a loss of diagnostic performance. The primary objective of the CONCENTRATE study is to prove the noninferiority of the enhancement effect of low-concentration contrast agents compared to a high-concentration contrast agent of the coronary artery and myocardium with coronary $\mathrm{CT}$ angiography.

Methods/Design: The CONCENTRATE study is a prospective, multicenter, noninferiority, randomized trial evaluating the enhancement effect of low-concentration contrast agents (270 and $320 \mathrm{mg}$ iodine/ml) compared with a highconcentration contrast agent (370 mg iodine/ml) in the coronary artery and myocardium of coronary artery CT angiography. The primary efficacy measurement is the enhancement of coronary arteries as measured in Hounsfield units. The target population comprises 318 patients with suspected coronary artery disease who have been referred for clinically indicated nonemergent coronary $C T$ angiography. Eligible participants are randomized for three different concentrations of the contrast agent in a 1:1:1 allocation ratio to one of three arms. The CONCENTRATE trial is a double-blind study, where the subjects and the outcome assessor are blinded to the concentration of the contrast agent used for coronary the CT angiography. Eight clinical sites in Korea are participating in this trial.
\end{abstract}

Discussion: The CONCENTRATE study will determine whether low-concentration contrast agents are able to provide diagnostic image quality in coronary CT angiography.

Trial registration: NCT02549794. Registered on 14 September 2015.

Keywords: Computed tomography, Coronary computed tomography angiography, CCTA, Coronary artery disease, CAD, Contrast agent, Radiation

\footnotetext{
* Correspondence: bchoi@yuhs.ac

'Department of Radiology and Research Institute of Radiological Science, Severance Hospital, Yonsei University College of Medicine, Seoul, Republic of Korea

${ }^{9}$ Department of Radiology, Severance Hospital, Yonsei University College of Medicine, 50-1 Yonsei-ro, Seodaemun-gu, Seoul 03722, Republic of Korea Full list of author information is available at the end of the article
} 


\section{Background}

Due to developments in computed tomography (CT) technology, cardiac CT has become very useful as a noninvasive examination technique in the diagnosis of obstructive coronary artery disease (CAD), and the accuracy has increased to more than $90 \%$ [1-5]. Specifically, cardiac CT plays a gatekeeper role in reducing invasive cardiac angiography implemented solely for the purpose of diagnosis [6]. However, cardiac CT also has disadvantages, particularly the exposure of patients to radiation and iodine contrast agent. Consequently, considerable effort has been devoted to identifying ways to reduce the radiation exposure and the amount of contrast agent used. A recently introduced method uses a combination of a scan with a low tube-based potential and iterative image reconstruction to reduce both the radiation dose and the amount of contrast agent used for coronary CT angiography [7]. According to recent studies of values from this combined method, the signal-tonoise ratio (SNR) and contrast-to-noise ratio (CNR), which represent image quality, are higher compared to the standard method $[8,9]$. The standard method utilizes a scan with higher tube potential according to patient body mass index (BMI) and image reconstruction by makeshift filtered-back projection under conditions using the same amount of contrast agent. Therefore, the amount of contrast agent can be reduced while achieving the same contrast effect due to the advantage of the increased effect of contrast enhancement provided by the low tube potential. Therefore, efficacy studies using low-concentration contrast agents along with low tube potential are being performed [10].

The CONCENTRATE study intends to prove that, compared to the combined method using the makeshift filtered-back projection image reconstruction and standard image acquisition according to BMI and with a standard high-concentration contrast agent, the image quality does not deteriorate as a result of the combination of a scan with low tube potential and the iterative image reconstruction method with low-concentration contrast agents.

\section{Study objectives \\ Primary objective}

The primary objective of the CONCENTRATE study is to determine the noninferiority of the contrast enhancement of cardiac CT with low-concentration contrast agents compared to that with high-concentration contrast agent.

\section{Secondary objective}

The secondary objective of the CONCENTRATE study is to determine the diagnostic accuracy of coronary $\mathrm{CT}$ angiography in the identification of anatomically obstructive
CAD with low-concentration contrast agent compared with invasive coronary angiography (ICA) as the reference standard and to compare it to the accuracy achieved with a high-concentration contrast agent.

\section{Primary hypothesis}

We hypothesized that the use of low-concentration contrast agent for coronary CT angiography and myocardial perfusion would not be inferior to the use of a highconcentration contrast agent in the enhancement effect in the coronary artery and in the myocardium.

\section{Methods/Design \\ Trial design}

The CONCENTRATE study is a prospective, multicenter, noninferiority, randomized trial evaluating the enhancement effect of low-concentration contrast agents consisting of 270 and $320 \mathrm{mg}$ iodine $/ \mathrm{ml}(\mathrm{mgI} / \mathrm{ml})$, compared with a high-concentration contrast agent that contains $370 \mathrm{mgI} / \mathrm{ml}$ in the coronary artery and myocardium in coronary artery CT angiography. The target population includes patients with suspected CAD who have been referred for clinically indicated nonemergent ICA. Eligible participants are randomized for three different contrast agent concentrations in a 1:1:1 allocation ratio to one of three arms of the trial. The CONCENTRATE trial is a double-blind study, where the subjects and the outcome assessor are blinded to the concentration of the contrast agent used for coronary CT angiography. Eight clinical sites in Korea are participating in this trial. Every clinical site requires the approval of the site's Institutional Review Board. The study protocol and the informed consent form should be approved by the Institutional Review Board at each participating site. A list of the Institutional Review Boards and the status of their respective approvals are provided in an additional file (see Additional file 1). Standard protocol items and organizational structures are provided as additional files (see Additional files 2 and 3, respectively). A flow chart of the study is provided in Fig. 1. The informed consent will be obtained from all participants (see Additional file 4).

\section{Participants}

Suitable participants include patients $\geq 20$ years of age who have requested coronary $\mathrm{CT}$ angiography to assess clinical disease. The exclusion criteria include the following: 1) subjects suspected of having myocardial infarction, unstable angina pectoris, or coronary artery disease; 2) subjects with heart attack within 40 days prior to the CT scan; 3) subjects with a diagnosed complicated heart anomaly; 4) $\mathrm{BMI}>35 \mathrm{~kg} / \mathrm{m}^{2}$; 5) serum creatinine $\geq 1.5 \mathrm{mg} / \mathrm{dl}$ of renal insufficiency; 6) pregnant subjects; 6) subjects with a history of hypersensitivity 


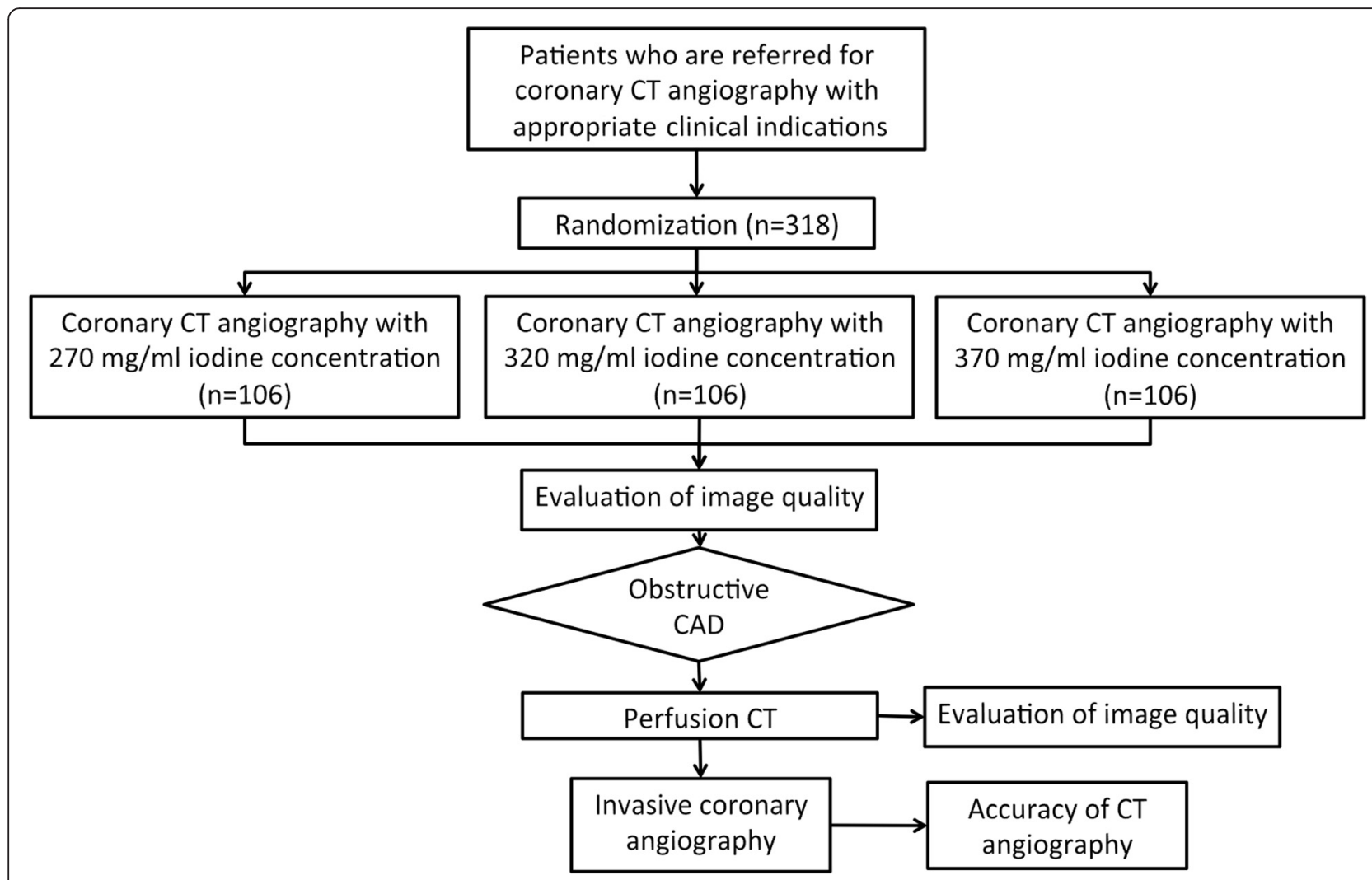

Fig. 1 CONCENTRATE study workflow. CT, computed tomography; CAD, coronary artery disease

reactions to contrast agents; 7) subjects with contraindications to the use of nitroglycerine; 8) subjects who plan to participate or enroll in other randomized clinical trials for cardiovascular disease; or 9) subjects with contraindications to the use of adenosine (e.g., bronchial asthma, 2-3 degree atrioventricular block, sick sinus syndrome, systolic blood pressure (SBP) less than $90 \mathrm{mmHg}$, recent prescribed history of dipyridamole, or hypersensitivity to adenosine) (Table 1). Patients who meet the selection criteria are registered by acquiring informed consent at the time an examination is ordered and during outpatient treatment by the investigators. Time schedule, interventions, assessments, and visits for participants are provided in a table (Table 2).

\section{Randomization}

All enrolled subjects are randomly assigned to one of three concentrations of contrast agent in a 1:1:1 ratio based on each trial site. We use concealed allocation and an adequate computer-generated allocation sequence to avoid selection bias. Thus, neither the patient nor the outcome assessor knows to which group the patient is allocated. Therefore, if unblinding is deemed necessary, any of the investigators, coordinators, or CT operators can provide the information of the contrast agent used. If the assessors of the outcome learn this information, they should report this on the corresponding case report form.

\section{Interventions}

Three different concentrations of contrast agent are randomly assigned to patients undergoing CT coronary angiography. A high tube potential is used for CT scans

Table 1 Inclusion and exclusion criteria

Inclusion criteria

- Adults at least 20 years old

- Subject who requested a coronary CT angiography to assess clinical disease

Exclusion criteria

- Subjects suspected of having myocardial infarction, unstable angina pectoris, or coronary artery disease

- Subjects who experienced heart attack within 40 days prior to the CT scan

- Subjects with a diagnosed complicated heart anomaly

- $\mathrm{BMl}>35 \mathrm{~kg} / \mathrm{m}^{2}$

- Serum creatinine $\geq 1.5 \mathrm{mg} / \mathrm{d}$

- Pregnant subjects

- Subjects with a history of hypersensitivity reaction to contrast agents

- Subjects with contraindications to the use of nitroglycerine

- Subjects who plan to participate or enroll in other randomized

clinical trials for cardiovascular disease.

- Subjects with contraindications to the use of adenosine (e.g. bronchial asthma, 2-3 degree AV block, sick sinus syndrome, SBP less than $90 \mathrm{mmHg}$, recent prescribed history of dipyridamole, or hypersensitivity to adenosine)

$C T$ computed tomography, BMI body mass index, SBP systolic blood pressure 
Table 2 Schedule of forms and procedures

\begin{tabular}{|c|c|c|c|c|c|}
\hline & Baseline & CT angiography & CT perfusion & Coronary angiography & Study \\
\hline & Visit 1 & Visit 2 & Visit 3 & Visit 4 & \\
\hline Screening & $x$ & & & & \\
\hline Demographic & $x$ & & & & \\
\hline Medical history & $x$ & & & & \\
\hline Randomization & $x$ & & & & \\
\hline Vital signs & & $x$ & & & \\
\hline Laboratory test & $x$ & & & & \\
\hline Vital signs 2 & & $x$ & & & \\
\hline CT angiography & & $x$ & & & \\
\hline Vital signs 3 & & & $x$ & & \\
\hline CT perfusion & & & $x$ & & \\
\hline Coronary angiography & & & & $x$ & \\
\hline Study evaluation & & & & & $x$ \\
\hline
\end{tabular}

with the high-concentration contrast agent $(370 \mathrm{mgI} /$ $\mathrm{ml}$ ), whereas a tube potential that is $20 \mathrm{kVp}$ lower is used with the low-concentration contrast agents (270 $\mathrm{mgI} / \mathrm{ml}$ and $320 \mathrm{mgI} / \mathrm{ml}$ ).

\section{Preparation of patients}

If participants do not have contraindications for the use of the nitroglycerine, they receive sublingual nitroglycerine before coronary CT angiography. If a participant's heart rate is equal to or greater than 60 beats per min, a beta-blocker is administered. If $>50 \%$ stenosis is apparent on the coronary CT angiography, participants will undergo stress perfusion CT. In these cases, nitroglycerine and beta-blockers are not used. Separate intravenous lines are secured for the injection of adenosine and contrast media.

\section{Contrast agents}

Three contrast agents with different iodine concentrations (Visipaque 270, iodixanol $270 \mathrm{mgI} / \mathrm{ml}$; GE Healthcare, Giles St Chalfont, United Kingdom vs. Visipaque 320, iodixanol $320 \mathrm{mgI} / \mathrm{ml}$; GE Healthcare, Giles St Chalfont, United Kingdom vs. Pamiray 370, iopamidol $370 \mathrm{mgI} / \mathrm{ml}$; Dongkook Pharma, Seoul, Korea) will be compared in this study. The participants are randomly assigned to different contrast agents in the same proportions. All contrast agents will be maintained by the same storage process, based on hospital systems, as used in general $\mathrm{CT}$ examinations. Each contrast agent will be administered via the antecubital vein of patients in the same triphasic injection. In the first phase, a $50-\mathrm{ml}$ bolus of contrast agent will be injected at $5 \mathrm{ml} / \mathrm{s}$. Then, $50 \mathrm{ml}$ of mixed saline with $30 \mathrm{ml}$ iodine and $20 \mathrm{ml}$ saline will be injected into patients at $5 \mathrm{ml} / \mathrm{s}$, followed by $40 \mathrm{ml}$ of saline chaser. The total injected volume of the contrast agent is $80 \mathrm{ml}$.

\section{Coronary CT protocol}

All coronary CTA studies are acquired with a multidetector CT scanner (Discovery HD 750; Gemstone Spectral Imaging, GE Healthcare, Milwaukee, WI, USA). During scanning, participants hold their breath and are still. To obtain better image quality for each contrast agent, scans are performed with different protocols depending on whether high- or low- concentration contrast agents are used. If a high-concentration contrast agent (iopamidol $370 \mathrm{mgI} / \mathrm{ml}$ ) is used, scans are conducted at $120 \mathrm{kVp}$ for higher BMI $(27<\mathrm{BMI}<35)$ patients and at $100 \mathrm{kVp}$ for lower BMI $(15<\mathrm{BMI} \leq 27)$ patients, with adjusted mAs (BMI-based tube potential selection). If a low-concentration contrast agent (iodixanol $270 \mathrm{mgI} / \mathrm{ml}$ or iodixanol $320 \mathrm{mgI} / \mathrm{ml}$ ) is used, scans are conducted $20 \mathrm{kVp}$ lower than in the BMI-based tube potential protocol: that is, at $100 \mathrm{kVp}$ for higher BMI and $80 \mathrm{kVp}$ for lower BMI. A beta-blocker is used for patients with a heart rate higher than 60 beats per minute. Scans initiate a bolus tracking method with a 4.8-s delay after reaching 100 Hounsfield units (HU) in the ascending aorta enhancement.

\section{Myocardial perfusion CT}

Stress myocardial perfusion CT will be performed in patients with at least one segment of greater than $50 \%$ stenosis on coronary CT angiography. A static CT perfusion protocol will be used, and stress will be induced by infusing $140 \mathrm{mcg} / \mathrm{kg} / \mathrm{min}$ adenosine under ECG monitoring for up to $6 \mathrm{~min}$. The randomly assigned contrast agent, which was used for coronary CT angiography in 
the patient, will be injected 4 min $30 \mathrm{~s}$ after the adenosine injection, using the same injection method for contrast agent and image acquisition settings as used for the coronary CT angiography with the only difference being an additional scan delay of $2 \mathrm{~s}$.

\section{Image reconstruction}

All images will be reconstructed using an iterative reconstruction algorithm at $50 \%$ adaptive statistical iterative reconstruction (ASIR, GE Healthcare, Waukesha, Wis, USA).

\section{Evaluation of radiation dose}

The dose-length product (DLP) from each shooting will be collected. The effective radiation dose (mSv) will be calculated using a conversion factor of $0.014 \mathrm{mSv} \mathrm{mGy}^{-1} \mathrm{~cm}^{-1}$.

\section{Retention}

This study does not follow the participants' outcome. Therefore, no specific plan has been developed to promote participant retention.

\section{Efficacy analysis \\ Primary efficacy analysis}

The primary efficacy measurement is the HU value of the coronary arterial lumen acquired from the comparison of the image quality from the three different protocols of contrast media in coronary CT angiography. The coronary artery is divided into 17 segments according to the modified American Heart Association (AHA) classification, and luminal enhancement is measured for each segment with avoidance of the borderline between the lumen and the vessel wall or epicardial tissue, artifacts, or calcification. Measurement is performed on three different points in each segment and the average value is used for the HU value of each segment. The mean HU value of left main artery and proximal right coronary artery will be compared between the different arms of the trial.

\section{Secondary efficacy analysis}

Qualitative evaluation of the image quality of the coronary artery will be performed. Two experienced observers will review all coronary $\mathrm{CT}$ images and score the image quality for each segment with a 4-point grading system on visual assessment: Grade 1, nondiagnostic; Grade 2, reduced image quality; Grade 3, nonlimiting artifacts; and Grade 4, complete absence of motion artifacts with good attenuation of the vessel lumen and clear delineation of the vessel walls with the additional ability to assess luminal stenosis.

Quantitative evaluation of image quality of perfusion $\mathrm{CT}$ will be performed. The myocardium will be divided into 16 segments according to AHA classification, and the $\mathrm{HU}$ value of each segment will be measured. Mean
HU value, SNR, and CNR of myocardial enhancement will be compared between the different arms of the trial.

Diagnostic accuracy of coronary CT angiography compared to invasive coronary angiography will be calculated. Segment-basis analysis, sensitivity, specificity, positive predictive value, negative predictive value, and accuracy of CT angiography for diagnosis of the presence of CAD defined by more than $50 \%$ diameter stenosis compared to invasive coronary angiography will be calculated and compared between the different concentrations of contrast agent by means of a generalized estimating equation based on a binary logistic model.

Quantitative evaluation of image quality of coronary artery will be performed on a per-vessel and persegment basis.

\section{Statistical methods}

\section{Sample size and power calculation}

The noninferiority margin was justified by an indirect confidence interval approach using the point estimate because the constancy assumption was not applicable in this trial. In a previous study [11], the reduction rate between iopamidol $370 \mathrm{mgI} / \mathrm{ml}$ and iodixanol $320 \mathrm{mgI} / \mathrm{ml}$ was $18 \%$ (from 439.96 to $362.06 \mathrm{HU}$ ), and we assumed that the $\mathrm{HU}$ value between the low-concentration and high-concentration groups would be the same. Consequently, we estimate $9 \%(0.5 \times(18 \%-0 \%))$ of the reported mean $\mathrm{HU}$ [10] to be the noninferiority margin, allowing a loss of less than $50 \%$ of the active control effect, which corresponds to a noninferiority margin of $51.26 \mathrm{HU}$ [12]. Clinically, 250-300 HU is considered sufficient enhancement for coronary angiography [13]. Therefore, a reduction of $51.26 \mathrm{HU}$ from $439.96 \mathrm{HU}$, which results in $388.7 \mathrm{HU}$, is clinically acceptable. For the mean contrast enhancement as an attenuation value of CT (HU) in the noninferiority test, we assumed same mean HU among the three groups and a standard deviation of 118.93 for the $\mathrm{HU}$ based on a previous study [10]. With these assumptions and a $10 \%$ dropout rate, 106 subjects per group are needed to obtain $80 \%$ statistical power with a corrected two-sided $\alpha \fallingdotseq$ $0.0167(=0.05 / 3)$.

\section{Primary statistical analysis}

Per-protocol analysis will be performed primarily; an additional intention-to-treat analysis will be also performed. The $98.33 \%$ confidence interval (CI) for the difference of mean contrast enhancement in the ROIs will be calculated. The noninferiority of the low-dose group compared to the high-dose group will be demonstrated if the lower bound of the two-sided $98.33 \%$ CI lies above the pre-specified noninferiority margin. Because missing values are expected for $5 \%$ or fewer of the participants, we have planned complete-case analysis for 
the primary analysis. To account for multiple observations per patient in secondary analysis, we will use a linear mixed model, including fixed effects for the group and random intercepts for the patient. Patients with a missing observation in some vessel or segment will be included in the per-vessel and per-segment analysis by using the linear mixed model. Analysis of covariance (ANCOVA) with BMI as a covariate will be used to compare the mean $\mathrm{HU}$ of the myocardium on static perfusion CT. Inter-reader agreement for the assessment of image quality will be evaluated using a linearly weighted kappa statistic. Analysis of variance (ANOVA) will be used to compare mean changes in heart rate before and after CT examinations among the three groups. To compare the diagnostic accuracy of invasive coronary angiography for diagnosis of significant coronary artery stenosis with more than $50 \%$ stenosis among the three groups, logistic regression analysis using a generalized estimating equation (GEE) will be used to account for the correlation among multiple segments within the same subject. All statistical analyses will be performed using SAS (SAS Ver. 9.2; SAS Institute, Cary, NC, USA) and two-sided $P$ values less than 0.05 will be considered statistically significant.

\section{Data management}

The data-coordinating center in Severance Hospital is collecting data through a secure Internet connection to the central server and monitoring the overall dataset. At the start of the trial, the monitors conducted a tutorial on the web-based data entry system and the image upload system. They will audit the overall quality and integrity of the data regularly every 6 months and, if necessary, contact the site investigator and coordinator to review and confirm the correctness of the data with source data in compliance with the protocol. The monitors will verify that all adverse events were documented in the correct format and are consistent with protocol definition. The monitor conducts the monitoring procedure independently from the investigators and the sponsor. The primary and secondary endpoints in this trial include only the image quality and not the patients' clinical outcomes. If the quality is not enough to determine significant coronary artery disease due to insufficient enhancement from a low-concentration contrast agent, repeat examination with a high-concentration contrast agent can be used at the site investigator's discretion. Therefore, the conduct of an interim analysis is not needed to evaluate any potentially important reasons to modify or discontinue the trial. Adverse effects and serious adverse events will be recorded in the case report form. A summary of adverse effects and serious adverse effects will be immediately forwarded the independent Institutional Review Board and local health authorities, according to local regulations.

\section{Discussion}

The CONCENTRATE study is a prospective, multicenter, noninferiority, randomized trial evaluating the enhancement effect of two low-concentration contrast agents compared with the high-concentration contrast agent recommended for sufficient opacification in coronary CT angiography, according to established guidelines [14].

The accuracy of coronary CT angiography in the diagnosis of coronary artery stenosis is affected by image quality, which is dependent on the CNR. To achieve a high CNR, high-concentration contrast agents are usually recommended and have been widely used in everyday clinical practice as a standard protocol [14]. According to a study that compared two contrast agents with different concentrations $(400 \mathrm{mgI} / \mathrm{ml}$ vs. $320 \mathrm{mgI} /$ $\mathrm{ml}$ ), coronary arterial enhancement was higher when the high-concentration contrast agent was used and higher enhancement levels were found to be associated with lower numbers of inadequately visualized segments [11]. However, in another study that compared four different concentrations (370, 350, 320, and $270 \mathrm{mgI} / \mathrm{ml}$ ), image quality grade was higher with low-concentration contrast agents, although high-concentration contrast agents showed greater vascular enhancement [15]. The fact that heart rate variability was lower with lowconcentration contrast agents, which were all isoosmolar agents, whereas the high-concentration contrast agents were all low-osmolar agents, might partially account for the higher image quality obtained with lowerconcentration-contrast agents in this study. The lowest enhancement of $369.1 \pm 85.4$ HU obtained with the lowest concentration of $270 \mathrm{mgI} / \mathrm{ml}$ in this study could be considered adequate because adequate intra-arterial enhancement for coronary CT angiography has been considered to require more than $250 \mathrm{HU}$ according to previous reports $[13,14]$.

In this regard, an effort to reduce the amount of iodine should be considered to reduce the probability of contrast-induced nephropathy in patients at risk, as long as diagnostic image quality is maintained $[16,17]$. When using low-concentration contrast agents, a combination of low tube potential and iterative reconstruction would be helpful for maintaining high vascular enhancement and image quality. A lower tube potential has the advantages of reducing radiation dose and improving image contrast. However, using a lower tube potential reduces $\mathrm{X}$-ray penetration and increases image noise. Iterative reconstruction is a solution for this problem because it improves image quality by reducing image noise. The combination of a lower tube potential and iterative 
image reconstruction does not result in a deterioration of image quality and diagnostic accuracy $[8,9]$. A single center study tested the feasibility of this combination with a low-concentration $(270 \mathrm{mgI} / \mathrm{ml})$ contrast agent compared to a high-concentration contrast agent (370 mg iodine $/ \mathrm{ml}$ ) in coronary CT angiography and demonstrated that a low-concentration contrast agent maintained the contrast enhancement without impairing image quality [10]. The CONCENTRATE trial has been designed to validate this finding as a multicenter study to limit possible bias and secure maximum generalizability.

The CONCENTRATE study will determine whether low-concentration contrast agents are able to provide diagnostic image quality on coronary $\mathrm{CT}$ angiography. Because the CONCENTRATE trial will only measure the coronary artery lumen and the myocardium in coronary CT angiography, the results of the CONCENTRATE trial should be further validated for other applications of cardiac CT, including in the evaluation of plaque, in-stent restenosis, and image-based fractional flow reserve. With expanding options for technology, such as feasibly scanning with $70 \mathrm{kVp}$, and more sophisticated iterative reconstruction methods, CT protocols should be further optimized to apply the results of the CONCENTRATE trial in the real world.

\section{Trial status}

Recruitment commenced in July 2015.

\section{Additional files}

Additional file 1: List of Ethics Committees and Status of Approval. List of the ethics committees of all eight participating centers and the status of approval of the study at the time of submission. (DOCX $13 \mathrm{~kb}$ )

Additional file 2: SPIRIT checklist 2013. (DOCX $52 \mathrm{~kb}$ )

Additional file 3: Organizational structure. (DOCX 86 kb)

Additional file 4: Model consent form. (DOCX $160 \mathrm{~kb}$ )

\section{Abbreviations}

AHA, American Heart Association; BMI, body mass index; CAD, coronary artery disease; CNR, contrast-to-noise ratio; $\mathrm{CT}$, computed tomography; ECG, electrocardiography; HU, Hounsfield Unit; ICA, invasive coronary angiography; SNR, signal-to-noise ratio

\section{Acknowledgements}

GE Healthcare is providing the funding for this study and provided the contrast agents (VISIPAQUE 270 and 320).

\section{Funding}

This investigator-initiated study is funded by GE Healthcare. The authors are solely responsible for the design and conduct of this study, analysis of the study data, drafting and editing of the paper, and the final content of the paper.

\section{Authors' contributions}

DJI, YHK, KSC, JWK, JIJ, YDW, HRK, MHC, and BWC participated in the study design and the process of patient enrollment. DJl, $\mathrm{KHH}$, and BWC drafted the manuscript. KHH participated in the design of the study, sample size calculation, and statistical analysis. All authors read and approved the final manuscript.

\section{Competing interests}

The authors declare that they have no competing interests.

\section{Ethics and dissemination}

If the need arises to modify an important aspect of the protocol, it will be reported and approval sought from the sites' Institutional Review Board. All records for participants will be stored in a secure place or/and in an accesslimited electronic file with password protection and subject identifier after removing personal information. These records will be kept during and after the trial according to legal requirements. The data-coordinating center will be allowed access to the final trial dataset, but access will not be allowed for the assessors of the trial endpoints. No compensation will be provided by the investigators for any post-trial care. All principal investigators at the participating sites are eligible for authorship, and no plan exists to use a professional writer or open the trial dataset to the public.

\section{Author details}

${ }^{1}$ Department of Radiology and Research Institute of Radiological Science, Severance Hospital, Yonsei University College of Medicine, Seoul, Republic of Korea. ${ }^{2}$ Department of Radiology, Chonnam National University Hospital, Chonnam University Medical School, Gwangju, Republic of Korea.

${ }^{3}$ Department of Radiology, Pusan National University Yangsan Hospital, Pusan National University School of Medicine, Pusan, Republic of Korea. ${ }^{4}$ Department of Radiology and Research Institute of Radiology, Asan Medical Center, University of Ulsan College of Medicine, Seoul, Republic of Korea. ${ }^{5}$ Department of Radiology, Seoul St. Mary's Hospital, The Catholic University of Korea, Seoul, Republic of Korea. ${ }^{6}$ Department of Radiology, Uijeongbu St. Mary's Hospital, Catholic University of Korea, Uijeongbu, Republic of Korea. ${ }^{7}$ Department of Radiology, Yeouido St. Mary's Hospital, Catholic University of Korea, Seoul, Republic of Korea. ${ }^{8}$ Department of Radiology, Bucheon St. Mary's Hospital, Catholic University of Korea, Bucheon, Republic of Korea. ${ }^{9}$ Department of Radiology, Severance Hospital, Yonsei University College of Medicine, 50-1 Yonsei-ro, Seodaemun-gu, Seoul 03722, Republic of Korea.

Received: 29 October 2015 Accepted: 13 June 2016

Published online: 15 July 2016

\section{References}

1. Budoff MJ, Dowe D, Jollis JG, Gitter M, Sutherland J, Halamert E, et al. Diagnostic performance of 64-multidetector row coronary computed tomographic angiography for evaluation of coronary artery stenosis in individuals without known coronary artery disease: results from the prospective multicenter ACCURACY (Assessment by Coronary Computed Tomographic Angiography of Individuals Undergoing Invasive Coronary Angiography) trial. J Am Coll Cardiol. 2008;52(21):1724-32. doi:10.1016/j.jacc. 2008.07.031.

2. Li M, Du XM, Jin ZT, Peng ZH, Ding J, Li L. The diagnostic performance of coronary artery angiography with 64-MSCT and post 64-MSCT: systematic review and meta-analysis. PLoS One. 2014;9(1):e84937. doi:10.1371/journal. pone.0084937.

3. Meijboom WB, Meijs MF, Schuijf JD, Cramer MJ, Mollet NR, van Mieghem CA, et al. Diagnostic accuracy of 64-slice computed tomography coronary angiography: a prospective, multicenter, multivendor study. J Am Coll Cardiol. 2008;52(25):2135-44. doi:10.1016/j.jacc.2008.08.058.

4. Miller JM, Rochitte CE, Dewey M, Arbab-Zadeh A, Niinuma H, Gottlieb I, et al. Diagnostic performance of coronary angiography by 64-row CT. N Engl J Med. 2008;359(22):2324-36. doi:10.1056/NEJMoa0806576.

5. Vanhoenacker PK, Heijenbrok-Kal MH, Van Heste R, Decramer I, Van Hoe LR, Wijns W, et al. Diagnostic performance of multidetector $C T$ angiography for assessment of coronary artery disease: meta-analysis. Radiology. 2007;244(2): 419-28. doi:10.1148/radiol.2442061218.

6. Shaw LJ, Hausleiter J, Achenbach S, Al-Mallah M, Berman DS, Budoff MJ, et al. Coronary computed tomographic angiography as a gatekeeper to invasive diagnostic and surgical procedures: results from the multicenter CONFIRM (Coronary CT Angiography Evaluation for Clinical Outcomes: an International Multicenter) registry. J Am Coll Cardiol. 2012;60(20):2103-14. doi:10.1016/j.jacc.2012.05.062. 
7. Funama $Y$, Taguchi $K$, Utsunomiya D, Oda S, Yanaga Y, Yamashita Y, et al. Combination of a low-tube-voltage technique with hybrid iterative reconstruction (iDose) algorithm at coronary computed tomographic angiography. J Comput Assist Tomogr. 2011;35(4):480-5. doi:10.1097/RCT. 0b013e31821fee94.

8. Park YJ, Kim YJ, Lee JW, Kim HY, Hong YJ, Lee HJ, et al. Automatic Tube Potential Selection with Tube Current Modulation (APSCM) in coronary CT angiography: comparison of image quality and radiation dose with conventional body mass index-based protocol. J Cardiovasc Comput Tomogr. 2012;6(3):184-90. doi:10.1016/j.jcct.2012.04.002.

9. Suh YJ, Kim YJ, Hong SR, Hong YJ, Lee HJ, Hur J, et al. Combined use of automatic tube potential selection with tube current modulation and iterative reconstruction technique in coronary CT angiography. Radiology. 2013;269(3):722-9. doi:10.1148/radiol.13130408.

10. Zheng M, Liu Y, Wei M, Wu Y, Zhao H, Li J. Low concentration contrast medium for dual-source computed tomography coronary angiography by a combination of iterative reconstruction and low-tube-voltage technique: feasibility study. Eur J Radiol. 2014;83(2):e92-9. doi:10.1016/j. ejrad.2013.11.006.

11. Becker CR, Vanzulli A, Fink C, de Faveri D, Fedeli S, Dore R, et al. Multicenter comparison of high concentration contrast agent iomeprol-400 with isoosmolar iodixanol-320: contrast enhancement and heart rate variation in coronary dual-source computed tomographic angiography. Invest Radiol. 2011;46(7):457-64. doi:10.1097/RLI.0b013e31821c7ff4.

12. James Hung HM, Wang SJ, Tsong Y, Lawrence J, O'Neil RT. Some fundamental issues with non-inferiority testing in active controlled trials. Stat Med. 2003;22(2):213-25. doi:10.1002/sim.1315.

13. Becker CR, Hong C, Knez A, Leber A, Bruening R, Schoepf UJ, et al. Optimal contrast application for cardiac 4-detector-row computed tomography. Invest Radiol. 2003;38(11):690-4. doi:10.1097/01.rli.0000084886.44676.e4.

14. Abbara S, Arbab-Zadeh A, Callister TQ, Desai MY, Mamuya W, Thomson L, et al. SCCT guidelines for performance of coronary computed tomographic angiography: a report of the Society of Cardiovascular Computed Tomography Guidelines Committee. J Cardiovasc Comput Tomogr. 2009; 3(3):190-204. doi:10.1016/j.jcct.2009.03.004

15. Honoris L, Zhong Y, Chu E, Rosenthal D, Li D, Lam F, et al. Comparison of contrast enhancement, image quality and tolerability in Coronary CT angiography using 4 contrast agents: a prospective randomized trial. Int J Cardiol. 2015;186:126-8. doi:10.1016/j.jicard.2015.03.240.

16. Katzberg RW, Barrett BJ. Risk of iodinated contrast material-induced nephropathy with intravenous administration. Radiology. 2007;243(3):622-8. doi:10.1148/radiol.2433061411.

17. Nyman U, Almen T, Aspelin P, Hellstrom M, Kristiansson M, Sterner G. Contrast-medium-Induced nephropathy correlated to the ratio between dose in gram iodine and estimated GFR in $\mathrm{ml} / \mathrm{min}$. Acta Radiol. 2005:46(8):830-42.

\section{Submit your next manuscript to BioMed Central and we will help you at every step:}

- We accept pre-submission inquiries

- Our selector tool helps you to find the most relevant journal

- We provide round the clock customer support

- Convenient online submission

- Thorough peer review

- Inclusion in PubMed and all major indexing services

- Maximum visibility for your research

Submit your manuscript at www.biomedcentral.com/submit

) Biomed Central 